Trans

continentales

\title{
Transcontinentales
}

Sociétés, idéologies, système mondial

\section{Démocratie et gouvernement local en Pologne}

Marie-Claude MAUREL et Maria HALAMSKA, Démocratie et gouvernement local en Pologne, Paris, CNRS éditions, 2006, 265 p.

Catherine Perron

\section{OpenEdition}

\section{Journals}

Édition électronique

URL : http://journals.openedition.org/transcontinentales/1021

DOI : 10.4000/transcontinentales.1021

ISBN : 978-2-7351-1565-5

ISSN : 1775-397X

Éditeur

Editions de la maison des sciences de l'homme

Édition imprimée

Date de publication : 1 juin 2007

Pagination : 177-179

ISBN : 978-2-200-92396-9

ISSN : 1950-1684

Référence électronique

Catherine Perron, « Démocratie et gouvernement local en Pologne», Transcontinentales [En ligne], 4 | 2007, document 14, mis en ligne le 11 mai 2011, consulté le 25 septembre 2020. URL : http:// journals.openedition.org/transcontinentales/1021; DOI : https://doi.org/10.4000/transcontinentales. 1021 
construction du nouvel ordre mondial. Modèle européen contre modèle américain? Soft power contre real-politik plus unilatéraliste? New Delhi n'estime pas devoir choisir entre ces deux options, mais entend jouer toutes ses cartes, en tirant parti du nouveau regard que portent sur elle aussi bien l'Union européenne que les États-Unis.

Tous ces ouvrages, au total, nourrissent un débat fécond entre Européens et Indiens, en mobilisant des experts d'horizons divers et largement renouvelés. Les index sont précieux. On regrette seulement que les responsables de ces publications se contentent de préfaces minimalistes : des introductions synthétiques, nourries des pluralités de points de vue avancés par ces pléiades d'auteurs, aideraient à caler les problématiques, et éclaireraient utilement la richesse des débats animant de telles rencontres.

Jean-Luc Racine

\section{Démocratie et gouvernement local en Pologne}

\section{Marie-Claude MAUREL \\ et Maria HALAMSKA, \\ Démocratie et gouvernement local en Pologne, Paris, CNRS éditions, 2006, $265 \mathrm{p}$.}

Il n'est plus possible d'ignorer la profonde fatigue démocratique (taux d'abstention élevés aux différents scrutins, vote pour des partis aux orientations radicales ou populistes, apathie politique des citoyens etc.) qui toucheles pays d'Europe centrale, dont la Pologne. Certains des maux qui affectent les systèmes politiques post-communistes sont connus: faiblesse des sociétés civiles, corruption endémique, vie politique marquée par la "sur-partisanisation" (les partis, seules organisations intermédiaires entre le citoyen et l'État, surévaluent en conséquence leur légitimité) et la «sur-parlementarisation" (concentration du jeu des pouvoirs entre l'État et le Parlement, au détriment des instances périphériques et des autres échelons politiques). Les études sur les travers politiques de ces pays ont souvent porté sur la scène politique nationale, sur les phénomènes politiques les plus visibles, les plus facilement appréhendables. En centrant l'analyse des transformations sur le niveau de gouvernement local en Pologne, l'ouvrage de Marie-Claude Maurel et Maria Halamska évite cet écueil, et fournit une profondeur de champ qui vient utilement enrichir les études de la transformation et la compréhension des phénomènes sociaux et politiques dans ce pays. Le changement de perspective - les pratiques locales éclairant les pratiques nationales dans un mouvement ascendant - permet de mieux saisir l'origine et la nature des maux identifiés au niveau central. Un autre grand mérite de cet ouvrage, qui le distingue d'autres travaux analogues, est de se fonder sur des enquêtes engagées dans la dernière décennie du communisme, et menées sur une période de vingt ans. Cela permet de rendre compte de manière fine de la présence du passé ou, autrement dit, des modes de dépendance de la voie de la transformation polonaise. Les auteures font prendre conscience de l'importance des ressources puisées dans les années 1980, montrant à quel point le changement engagé en 1989 en Pologne prend racine dans les dernières années du régime communiste.

À travers une analyse du changement social survenu depuis le début des années 1980 dans trois petites communes rurales (représentatives de la majorité des communes du pays et situées dans trois régions de Pologne différentes) 
Marie-Claude Maurel et Maria Halamska se sont attachées à «recueillir, dans leur expression concrète, les faits de la "transition" annoncée» (p. 12.), opérant des allers-retours incessants entre la dynamique de transformation nationale et son expression locale. Si la première partie, intitulée «Du monopole au partage du pouvoir», est consacrée à la dimension institutionnelle du processus de démocratisation, c'est avant tout pour fournir une image du cadre dans lequel s'opère l'apprentissage des nouvelles règles du jeu par les acteurs locaux. Car c'est à ce dernier processus que les auteures s'intéressent avant tout. En effet, dans le temps de latence entre l'introduction de nouvelles institutions et l'appropriation de leurs règles de fonctionnement par les acteurs, s'opère tout un processus d'expérimentation et d'apprentissage, crucial à la construction démocratique et à son enracinement. Dans une deuxième partie, l'analyse porte sur la «recomposition des scènes politiques locales» pour finir dans une troisième partie par traiter de l'enjeu que représente l'émergence d'une nouvelle gouvernance à cette échelle. Le plan de l'ouvrage témoigne du glissement progressif, au fil du temps, des enjeux politiques relatifs au renouvellement vers ceux de la gouvernance locale, autrement dit du passage du défi que représentait la rupture avec le régime précédent à celui constitué par l'usage des nouvelles règles et compétences dont ont été investis les conseils municipaux à la suite du rétablissement de l'autonomie locale.

Les auteures opèrent un large tour d'horizon de la transformation du gouvernement local, allant de la restauration de l'autonomie à la base et de la transformation des cadres institutionnels qui l'accompagne, aux dynamiques politiques (partisanes) dont les élections constituent le point d'orgue, en passant par les acteurs politiques, la formation des élites, etc. S'y ajoute une description factuelle, nourrie de ce que fut la réalité de la transformation dans les trois communes étudiées, et ce faisant pour quantité de Polonais : liberté et possibilité de prendre son destin en main certes, mais dans un contexte d'appauvrissement, de repli sur la cellule familiale et de forte mise à mal du tissus social local. Les exemples concrets éclairent les raisons profondes du désenchantement démocratique en même temps qu'ils donnent une idée de l'ampleur des défis posés à ces communautés locales par le post-communisme. Malgré une vision souvent critique du mode de gouvernance locale, on ne peut s'empêcher d'éprouver de l'empathie pour ces acteurs en première ligne dans la gestion des difficultés de la transformation, démunis de ressources au départ et qui donnent le sentiment d'avoir été très largement abandonnés par les échelons supérieurs. Un point sur lequel on aurait aimé en savoir plus, d'ailleurs.

Si l'étude rend palpable l'ampleur des efforts que doivent fournir les acteurs du changement, pour extirper leur communauté locale du régime précédent et surmonter les pratiques et arrangements institutionnels traditionnels dans lesquels fonctionnaient les acteurs sociaux par le passé, on peut toutefois regretter que peu d'attention soit finalement portée au discours de ces acteurs, aux efforts qu'ils fournissent (ou non) en vue d'interpréter la trajectoire de leur commune au regard des transformations générales, à leur manière d'envisager l'avenir, d'interpréter le passé. En effet, on peut supposer que face à l'hébétude généralisée, à la disparition des institutions et des procédures qui jusquelà régissaient le quotidien, des règles 
qui ordonnaient les vies, et aux difficultés matérielles de la transformation, la production d'un discours fournissant du sens à l'action est un puissant facteur de mobilisation qui, en conférant du sens au changement, aide à surmonter le sentiment d'aliénation éprouvé par une grande partie de la population.

Pour finir, si le choix de la Pologne pour mener une telle enquête semble pleinement justifié - c'est le pays le plus peuplé de l'ancien bloc de l'Est et qui de ce fait a le plus de poids parmi les nouveaux adhérents à l'Union européenne, un pays rural à l'histoire nationale complexe -, on peut cependant regretter l'absence de démarche comparative ou de remise en perspective dans le cadre centre-européen, qui aurait permis de faire la part des spécificités polonaises et des traits plus généraux du post-communisme.

Catherine Perron Centre d'études et de recherches internationales (CERI) 